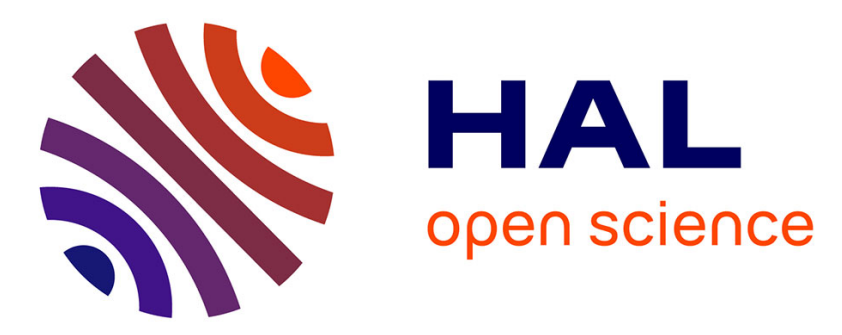

\title{
A thin and conformal metasurface for illusion acoustics of rapidly changing profiles
}

\author{
Marc Dubois, Chengzhi Shi, Yuan Wang, Xiang Zhang
}

\section{To cite this version:}

Marc Dubois, Chengzhi Shi, Yuan Wang, Xiang Zhang. A thin and conformal metasurface for illusion acoustics of rapidly changing profiles. Applied Physics Letters, 2017, 110 (15), pp.151902. 10.1063/1.4979978. hal-02145702

\section{HAL Id: hal-02145702 https://hal.science/hal-02145702}

Submitted on 3 Jun 2019

HAL is a multi-disciplinary open access archive for the deposit and dissemination of scientific research documents, whether they are published or not. The documents may come from teaching and research institutions in France or abroad, or from public or private research centers.
L'archive ouverte pluridisciplinaire HAL, est destinée au dépôt et à la diffusion de documents scientifiques de niveau recherche, publiés ou non, émanant des établissements d'enseignement et de recherche français ou étrangers, des laboratoires publics ou privés. 


\section{A thin and conformal metasurface for illusion acoustics of rapidly changing profiles}

Marc Dubois, Chengzhi Shi, Yuan Wang, and Xiang Zhang

Citation: Appl. Phys. Lett. 110, 151902 (2017); doi: 10.1063/1.4979978

View online: http://dx.doi.org/10.1063/1.4979978

View Table of Contents: http://aip.scitation.org/toc/apl/110/15

Published by the American Institute of Physics

\section{Articles you may be interested in}

Bifunctional acoustic metamaterial lens designed with coordinate transformation

Appl. Phys. Lett. 110, 113503113503 (2017); 10.1063/1.4978689

Optically read displacement detection using phase-modulated diffraction gratings with reduced zeroth-order reflections

Appl. Phys. Lett. 110, 151104151104 (2017); 10.1063/1.4979541

Experimental prototype of a spin-wave majority gate

Appl. Phys. Lett. 110, 152401152401 (2017); 10.1063/1.4979840

Three-terminal quantum-dot thermal management devices

Appl. Phys. Lett. 110, 153501153501 (2017); 10.1063/1.4979977

Dynamics of transient microbubbles generated by fs-laser irradiation of plasmonic micropyramids Appl. Phys. Lett. 110, 153102153102 (2017); 10.1063/1.4979886

Broadband metamaterial for optical transparency and microwave absorption

Appl. Phys. Lett. 110, 143511143511 (2017); 10.1063/1.4979543
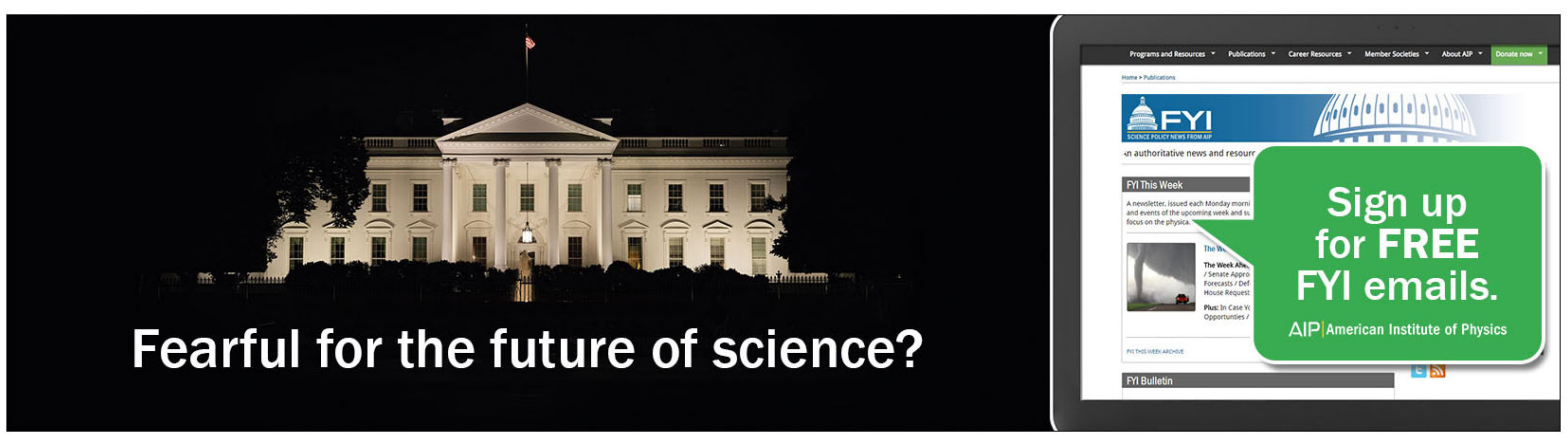


\title{
A thin and conformal metasurface for illusion acoustics of rapidly changing profiles
}

\author{
Marc Dubois, ${ }^{1, a)}$ Chengzhi Shi, ${ }^{1, a)}$ Yuan Wang, ${ }^{1}$ and Xiang Zhang ${ }^{1,2, b)}$ \\ ${ }^{1}$ NSF Nano-scale Science and Engineering Center (NSEC), University of California, Berkeley, \\ 3112 Etcheverry Hall, Berkeley, California 94720, USA \\ ${ }^{2}$ Materials Science Division, Lawrence Berkeley National Laboratory, 1 Cyclotron Road, Berkeley, \\ California 94720, USA
}

(Received 25 October 2016; accepted 29 March 2017; published online 10 April 2017)

\begin{abstract}
Recently developed metasurfaces have been used for surface engineering applications. However, the ability to cloak or mimic reflective surfaces with a large in-plane phase gradient remains unexplored. One major challenge is that even with a small incidence angle, the strong acoustic impedance variation induced by the random height profile creates additional scattering and increases the complexity of the analysis and design of the metasurface. Here, we introduce an acoustic metasurface with 1/12 wavelength thickness to realize an acoustic carpet cloak for a randomly rapid-change surface and a virtual acoustic diffuser from a flat surface using a set of Helmholtz resonators. The limitation of the metasurface for large phase gradient application is explored based on a nonlocal model that considers the contributions from neighboring surface profiles. This study extends the integration of smart acoustic surface and may find applications of surface engineering such as in architectural acoustics. Published by AIP Publishing.
\end{abstract}

[http://dx.doi.org/10.1063/1.4979978]

Absorbers and diffusers are essential elements to control echoes in architectural acoustics. In several applications, acoustic diffusers are preferred because absorbers remove sound energy from the environment, whereas they redistribute the acoustic energy in order to avoid strong dispersion and reverberating paths. ${ }^{1,2}$ Traditional diffusers are heavy, bulky wavelength scale structures with complex geometries and are therefore difficult to integrate in existing environments. Recently developed metasurfaces provide the ability to control waves with a single layer of deep-subwavelength elements. Owing to their ultra-thin dimension, metasurface elements can be conformally attached to an arbitrary shape to virtually reproduce the acoustic response of another geometry. In optics, metasurfaces give the freedom of modulating the phase of light to realize beam steering, ${ }^{3-5}$ ultrathin optical skin cloak, ${ }^{6}$ smaller optical devices for advanced digital products, ${ }^{7,8}$ and holograms. ${ }^{9-11}$ The subwavelength metasurface concept is especially important for acoustic wave manipulations as the wavelength is much larger than optics and the size constraint is critical. Coiled space elements were developed as building blocks of the acoustic metasurface to modulate the sound phase by increasing the effective path length and have been used for beam steering. ${ }^{12-21}$ However, the thickness of coiled space based metasurfaces is difficult to shrink down at less than quarter wavelength because a large intrinsic loss will result from viscosity in the extreme narrow channels. ${ }^{22}$ Another type of acoustic metasurface is formed by membranes decorated with masses, which were used to realize simultaneous negative density and bulk modulus ${ }^{23}$ and an acoustic perfect absorber. ${ }^{24} \mathrm{~A}$ thin acoustic cloak was also proposed theoretically using

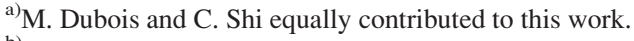

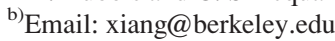

membrane type metasurfaces ${ }^{25,26}$ and was demonstrated by Helmholtz resonators with varying cavity volumes. ${ }^{27} \mathrm{Up}$ to date, the design of metasurfaces is typically assuming a continuous phase profile.

In this paper, we extend the previous design of the metasurface carpet cloak to investigate the effect of the large in-plane (rapidly changing) phase gradient on the cloaking performance. In the first order approximation, the carpet cloaking requires each metasurface element to provide a phase shift $\Delta \phi(x)=-2 k h(x) \cos \theta$, where $k$ is the wave number, $h(x)$ is the height profile of the structure to be cloaked (Figure 1(b)), and $\theta$ is the incident angle. ${ }^{6,25-27}$ This phase shift compensates the path difference due to the geometry of the object. Consequently, the reflected wavefront from the structure decorated with the metasurface is flat, and the object becomes invisible for the acoustic wave, as shown in Figure 1(a). On the other hand, metasurface elements can be used to make a flat surface to be a virtual diffuser for the acoustic wave. In Figure 1(c), we apply a metasurface with the exact opposite phase modulation $\Delta \phi^{\prime}(x)=2 k h(x) \cos \theta$ to recreate the scattering pattern of the object observed in Figure 1(b) from a flat surface. As sketched in Figure 1(d), previous studies in optics and acoustics demonstrated the ability to cloak structures with small continuous in-plane phase variation. ${ }^{6,25-27}$ On the other hand, Figure 1(e) presents a structure where the pillars' heights are chosen randomly and vary dramatically from site to site. During this random process, sharp welllike structures are created among the pillars, as detailed in Figure 1(f). These wells have very narrow width leading to an impedance mismatch that did not exist in the smooth gradient structure. We are able to analytically derive the reflection coefficient $R$ obtained from the well structure sketched in Figure 1(f) 
(a) Cloaking

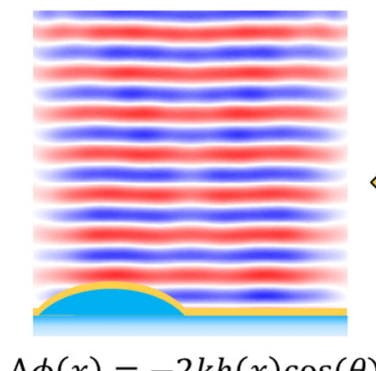

$\Delta \phi(x)=-2 k h(x) \cos (\theta)$

(d)

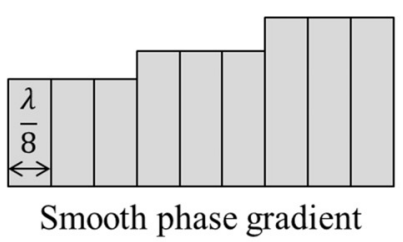

(f)

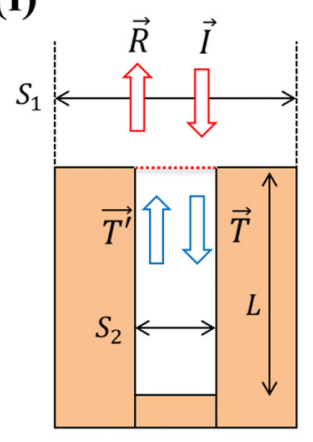

(b) Real Scattering

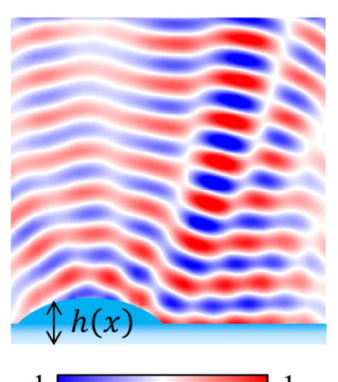

$-1 \longleftarrow 1$

Normalized pressure $(\mathrm{Pa})$

\section{(c) Virtual Scattering}

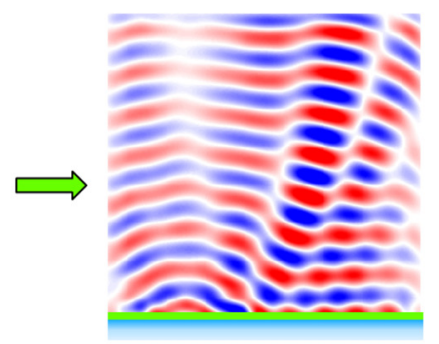

$\Delta \phi^{\prime}(x)=2 k h(x) \cos (\theta)$

(e)

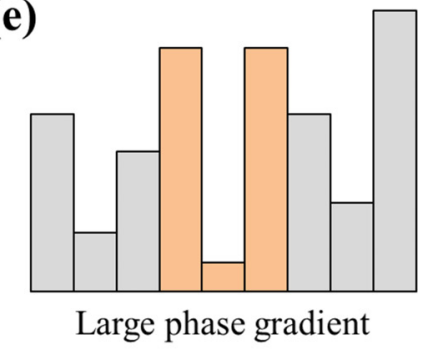

FIG. 1. Acoustic surface engineering for reflected waves. (a) The actual reflected field of a simple bump. The scattering pattern allows the acoustic wave to detect the existence of the bump. (b) Acoustic cloaking of a bump with the metasurface. The metasurface modulates the phase to flatten the reflected wavefront. Thus, the bump is invisible through the acoustic wave. (c) The acoustic diffuser formed by the metasurface on a flat surface. With the inverse phase mask of the cloak in (b), the scattering pattern in (a) is created. Therefore, the flat surface with the decorated metasurface mimics the bump structure, resulting in a virtual acoustic diffuser. ((d) and (e)) Sketch of structure inducing, respectively, small and large in plane phase gradients. (f) Detailed view of the well structure formed in (e) because of the height variation. (g) Calculated additional phase and amplitude in the reflection $R$ from the well structure for a ratio $m=S_{1} / S_{2}=3$ as a function of the well depth $L$ normalized by the incident wavelength.

$$
\frac{R}{I}=\frac{m-1+e^{-i 2 k L}(m+1)}{m+1+e^{-i 2 k L}(m-1)},
$$

with $m=S_{1} / S_{2}$ and $T=T^{\prime} e^{-2 i k L}$. From this expression, we can show that the amplitude of this reflection coefficient $R$ is always unity as no energy is stored in the well. However, Figure $1(\mathrm{~g})$ presents the additional phase term $\Delta \phi=\arg \left(\frac{R}{I}\right)$ $-2 k L$ acquired by the reflected field in the presence of the well for a ratio $m=3$. It is clear that the impedance mismatch provides an additional phase term to the reflected wavefront. The goal of this work is to investigate if this additional scattering can be compensated (or reproduced) by the intuitive phase shift profile formula given earlier.

We use an ultrathin acoustic metasurface formed by Helmholtz resonators for surface scattering designs. These Helmholtz resonators are ultra small compared with the wavelength, forming a metasurface with $1 / 12$ wavelength thickness (Figure 2(a)). The phase of the reflected wave from the resonators is controlled by the neck diameter (Figure 2(b)). Finite element method simulations, including the air viscosity model and thermoviscous boundary layer effects ${ }^{22}$ (see supplementary material, Section 2), show that the reflection phase can be tuned over the entire phase range, enabling the complete modulation of the reflected wavefront. In our experiment, we select six Helmholtz resonators with increasing neck diameters, resulting in six different reflection phases at $2.9 \mathrm{kHz}: 60,120,180,240,300$, and $360^{\circ}$. The measured resonances of the $3 \mathrm{D}$ printed resonators deviate slightly from the designed frequencies as a result of the fabrication error (Figure 2(c)). However, this disagreement only leads to a deviation within $20^{\circ}$ from the designed value in the reflection phase which is reasonable for our experimental setup. $20 \%$ of the incident amplitude is absorbed due to the material loss of the resonator units. Four cylindrical slots are printed on the back of each resonator such that they fit on LEGO bricks. This mechanism enables straightforward reconfigurations of metasurfaces formed by the Helmholtz resonators on structured surfaces built from standard LEGO bricks.

A recent study on the acoustic metasurface demonstrated a thin carpet cloak by modulating the reflection phase with a thicker design of Helmholtz resonators. ${ }^{27}$ Our design only changes the neck diameter, allowing the realization of deep subwavelength elements $(\lambda / 7.5 \times \lambda / 7.5 \times \lambda / 12)$. Moreover, the possibility of developing an ultrathin carpet cloak for a complex random structure as shown in Figure 3(a) remains an open question in both acoustics and electromagnetics. As 


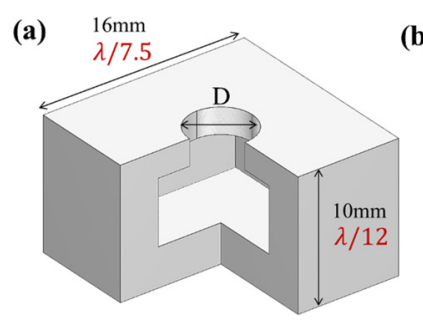

(b)
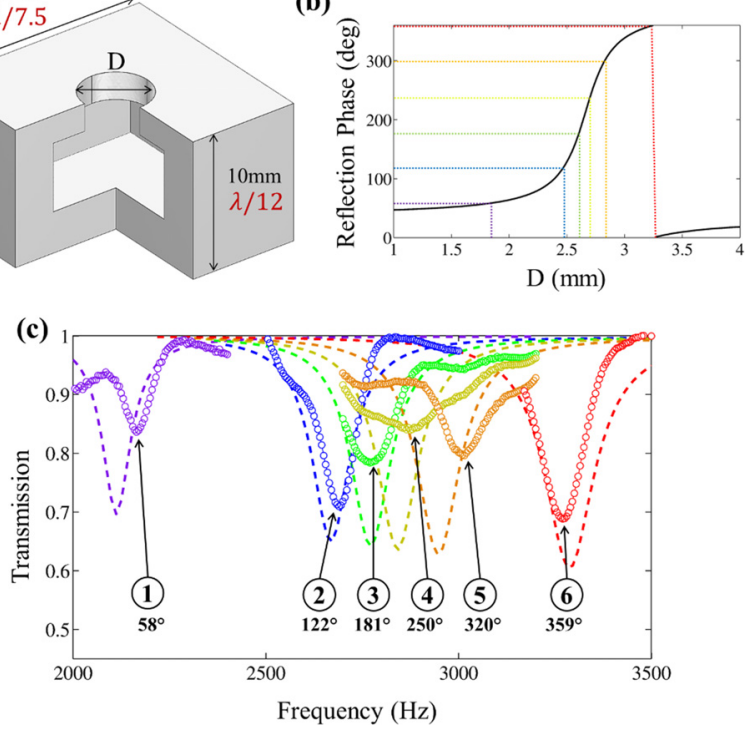

FIG. 2. Unit cells of the metasurface used for acoustic surface engineering. (a) A unit cell formed by a Helmholtz resonator. The unit cell has dimensions of $16 \mathrm{~mm} \times 16 \mathrm{~mm} \times 10 \mathrm{~mm}$ with a $10 \mathrm{~mm} \times 10 \mathrm{~mm} \times 5 \mathrm{~mm}$ cavity and a cylindrical neck, whose diameter is $\mathrm{D}$ and height is $2 \mathrm{~mm}$. (b) Numerically calculated relation between the reflection phase and the neck diameter at $2.9 \mathrm{kHz}$ with COMSOL (see supplementary material, Section 2). By varying the neck diameter, the resonance of the Helmholtz resonator is shifted. For the same working frequency, this resonance shift results in a different reflection phase. Six different neck diameters are chosen to realize 60 , $120,180,240,300$, and $360^{\circ}$ reflection phase shifts as marked by the dotted lines. (c) Measured (dotted lines) and numerically calculated (dashed lines) resonances of the six selected Helmholtz resonators. Viscosity loss and thermoviscous boundary layers are considered in the COMSOL simulations (see supplementary material, Section 2). The resonances are characterized by the dips of the transmission in an acoustic tube waveguide with a corresponding Helmholtz resonator assembled on the side wall of the waveguide. The resonant frequencies of the six resonators shift from the designed values as a result of fabrication errors. The resulted reflection phase modulations are $4 \%$ within the desired values.

discussed above, the abrupt changes of height in the structure induce not only a large phase gradient but also variations of the acoustic impedance, resulting in an additional phase term in the reflected field. In our experiment, we randomly choose the height of each column between zero and one wavelength. The width of these columns is $1 / 7.5$ wavelength, allowing us to create a diffuser with the large phase gradient (Figure 3(a)). The measured scattering of the normal incident plane wave reflected from the complex random structure at $2.9 \mathrm{kHz}$ is shown in Figure 3(c). With the metasurface formed by our Helmholtz resonators providing a local phase shift $\Delta \phi(x)=-2 k h(x)$ (for small incident angle) canceling the reflection phase variation solely due to the height change of the structure, the scattering is eliminated and a planar wavefront is observed in the reflection (Figure 3(d)). This scattering cancellation can be accomplished between $2.8 \mathrm{kHz}$ and $3 \mathrm{kHz}$ with the $3 \mathrm{D}$ printed Helmholtz resonators (see supplementary material, Section 3 ). Therefore, this metasurface design has the ability to cancel simultaneously the scattering from the surface roughness and impedance mismatch. As shown in Fig. 1(f), the phase shift between $T$ and $T$ ' comes from the distance $2 L$ traveled by the acoustic field. In the case of the decorated structure, one Helmholtz resonator is placed at the bottom of the well; this resonator is specifically chosen to add a (a)

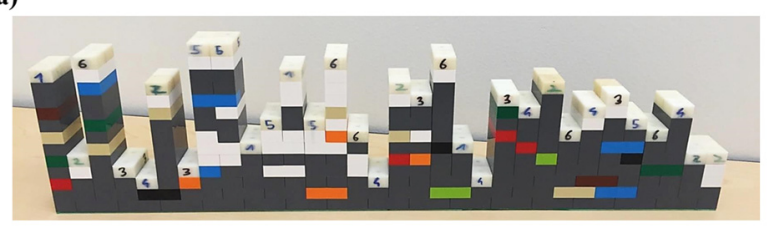

(b)

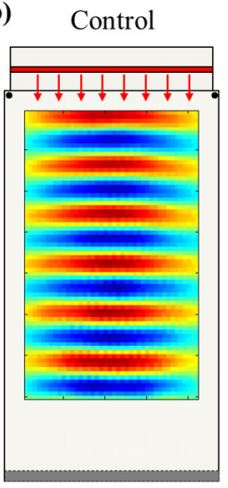

(c)

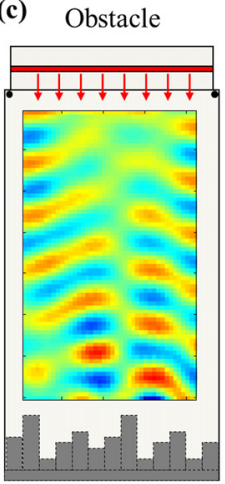

(d)

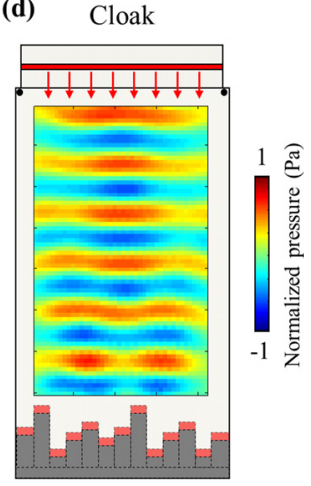

FIG. 3. Acoustic cloaking with the metasurface. (a) The actual structure used in experiments. The complex random structure is constructed from LEGO bricks. This complex random structure is decorated by $3 \mathrm{D}$ printed Helmholtz resonators forming an ultrathin metasurface for acoustic surface engineering. (b) Measured reflection field of normal incidence from a flat reflection surface at $2.9 \mathrm{kHz}$. A flat wavefront is observed. This scan serves as the control experiment for comparison with the cloaking experiment. (c) Measured reflection field of normal incidence from a complex random structure at $2.9 \mathrm{kHz}$. The reflection field presents the multi-scattering pattern as a result of the height variation of the reflection surface and impedance mismatch due to the variation of cross-section area. (d) Measured reflection field of normal incidence from the complex random structure decorated with cloaking metasurface at $2.9 \mathrm{kHz}$. The cloaking metasurface modulates the reflective surface by manipulating the reflection phase such that the height of each column is reduced virtually to the based surface (flat surface in (a)). Thus, the acoustic wave detects a flat surface and reflected with the flat wavefront. Consequently, the complex random structure is cloaked by the metasurface.

phase term $\Delta \phi=-2 k L$ to the field $T$. Therefore, we obtain $T=T^{\prime} e^{-2 i k L-i \Delta \phi}$, and we can insert this in Eq. (1)

$$
\frac{R}{I}=\frac{m-1+e^{-i 2 k L-i \Delta \phi}(m+1)}{m+1+e^{-i 2 k L-i \Delta \phi}(m-1)} .
$$

As $\Delta \phi=-2 k L$, Eq. (2) is simplified as $R=I$ which is what we would expect if the wave was reflected by a flat hard wall. This analysis and the experimental result demonstrate that metasurfaces with phase shift only depending on the local elevation can be used to cloak complex random structures with a large in-plane phase gradient. As of the incident angle dependence, numerical simulations show that our metasurface works until $+/-20^{\circ}$. This is expected from the local phase shift equation $\Delta \phi(x)=-2 k h(x) \cos \theta$ which is a second order function for small $\theta^{27}$ (see supplementary material, Section 4).

A flat surface can also be transformed into a diffusive surface for acoustic waves using metasurfaces with appropriate combinations of the unit cells. This transformation of mirror surfaces into diffusive surfaces is the inverse problem of cloaking. One can anticipate that it should be achieved by metasurfaces (for small incident angle) with the opposite local phase shift $\Delta \phi^{\prime}(x)=2 k h^{\prime}(x)$, where $h^{\prime}$ is the local height of the virtual object. In our experiment, we reproduce the scattering pattern of a circular bump (Figure 4(a)) from a 
flat metasurface at $2.9 \mathrm{kHz}$. By applying the calculated phase shift, a virtual acoustic object is created, and the same cylindrical wavefront is obtained in the reflection (Figure 4(b)). Therefore, the flat surface is transformed into a diffusive surface by our metasurface.

It is interesting to ask if we can transform the flat surface into the complex random surface shown in Figure 3(a). By applying the same strategy, a diffusive phase mask is applied on a flat surface. Surprisingly, the measured reflection shown in Figure 4(d) is different from that in Figure 4(c) for the complex random structure. Although the metasurface shifts the reflection phase to mimic the height variation, the abrupt (a) Obstacle

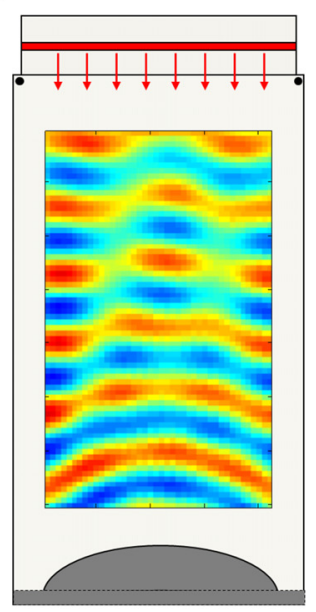

(c)

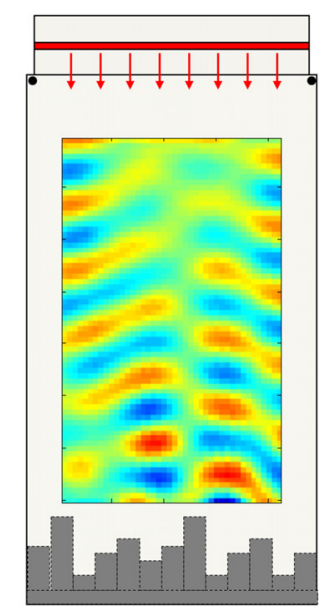

(b) Virtual Scattering

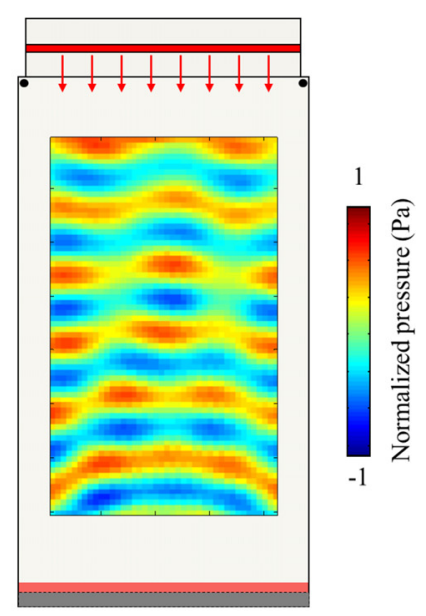

(d) Virtual Scattering

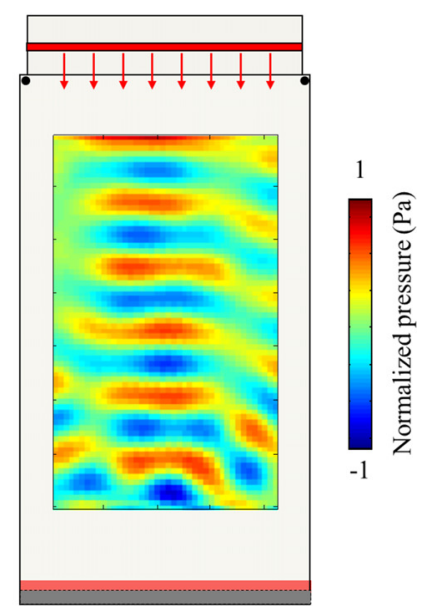

FIG. 4. An acoustic diffuser created by the metasurface. (a) Measured reflection field of normal incidence from a circular bump at $2.9 \mathrm{kHz}$. The circular bump results in wave scattering that diffuses the incident acoustic wave in all directions. (b) Measured reflection field of normal incidence from a flat reflection surface decorated with the diffusive metasurface. The metasurface modulate the reflection phase such that the local reflection surface presents a virtual height variation identical to the circular bump in (a). This virtual height variation results in the same scattering pattern that diffuses the acoustic wave in all directions. Thus, the metasurface creates an acoustic diffuser from a flat reflection surface. (c) The measured reflection field of normal incidence from a complex random structure is shown in Figure 3(b). The multi-scattering pattern is a result of the combination of the reflections due to the rough surface and impedance mismatch. (d) The measured reflection field of normal incidence from a flat reflective surface decorated with the inverse phase mask used for cloaking in Figure 3(c). The scattering pattern is different from the complex random structure. Thus, the inverse phase mask cannot recreate the scattering of the complex random structure. changes of impedance described previously are not captured in this simple relation. We can use the well structure (Figure $5(a))$ as a model to demonstrate this limitation. In order to reproduce the reflection $R$ observed in Figure 5(a), we need to determine the amplitude $(A)$ and phase $(\phi)$ that our element has to reflect in Figure 5(b). Assuming that each side of the well reflects perfectly the incident wave, we obtain the following relation

$$
A e^{i \phi}=m\left(\frac{R}{I}-1\right)+1,
$$

with $m=S_{1} / S_{2}$. The amplitude and phase of this field are represented in Figure 5(c) as a function of the well depth $L$ in the case $m=3$. First, we can see that the phase is no longer a linear function of the well depth. Second, and more importantly, the amplitude $A$ is larger than unity and needs to be modulated as a function of $L$. We have numerically checked the validity of Eq. (3) in Figure 5(e). Three elements highlighted in green are used to reproduce the field reflected by the triple well structure shown in Figure 5(d). The amplitude and phase of the field emitted by these elements are chosen with respect to the well depth (Figure 5(c)). However, the experimental demonstration of this result is hindered by the fact that our metasurface cannot modulate the reflected amplitude. The Helmholtz resonators are used as phase elements, whose amplitude response is constant and cannot exceed unity. This result explained why the linear (a)

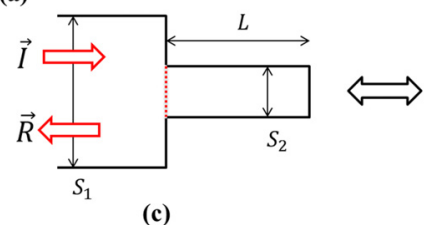

(c) (b)

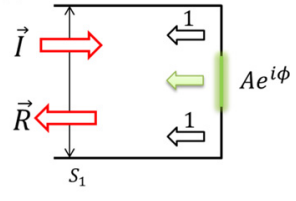

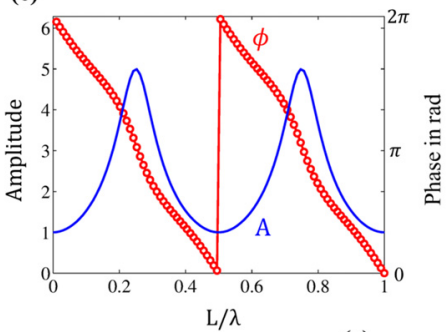

(d)

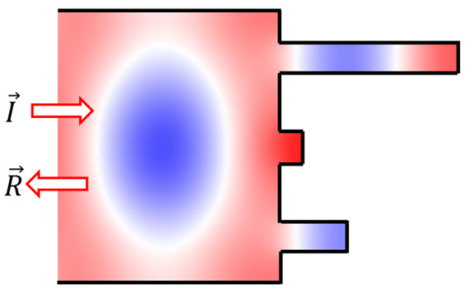

(e)

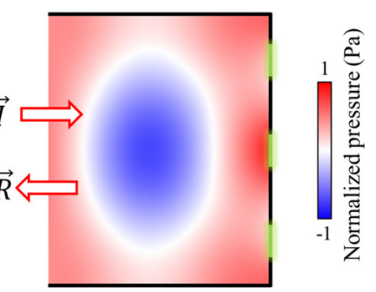

FIG. 5. Inverse phase mask for the large in-plane phase gradient. (a) Sketch of the well like structure emerging from the large in-plane phase gradient objects. (b) Sketch of the virtual diffuser configuration. The amplitude and phase of the purple element are chosen such that the new structure presents the same reflection $R$ as in (a). (c) Analytically calculated amplitude $A$ and phase $\phi$ obtained from (Eq. (3)) with $m=3$ as a function of the well depth $L$ normalized by the incident wavelength. (d) Numerical simulation of the field reflected by a triple well structure at $2.9 \mathrm{kHz}$ with the experimental dimensions (width of 7.5 ). (e) Reproduction of the reflected field with 3 active elements is highlighted in green. Amplitude and phase are taken from (c) to the well depth. 
relation between the phase and the height of the structure fails when the height varies at a deep subwavelength scale. Recent works have proposed an alternative approach based on holography to control the acoustic field propagation. ${ }^{28,29}$ However, the phase retrieval technique used to obtain the phase mask is only achieved with conditioned numerical iterations.

In conclusion, an ultrathin metasurface formed by Helmholtz resonators with varying neck diameters is realized to modulate the reflection phase. An ultrathin acoustic cloak for a complex random structure with abrupt height variation is realized. The inverse modulation of the reflection phase with reconfigured metasurface transforms flat surfaces into diffusive structures with a smooth phase gradient. The linear relation linking the phase and geometric profiles works for cloaking applications independently from the structure but fails to recreate virtual diffusers with the large in-plane phase gradient. This experimental and analytical study of the ultrathin acoustic metasurface for large in-plane phase gradient applications provides a deep understanding of reflective surface engineering, which is critical for smart and architectural acoustic designs.

See supplementary material for more details on the experimental apparatus. Additional results and discussions concerning air viscosity, incident angle dependence, and bandwidth are also provided.

This research was supported by the Office of Naval Research (ONR) MURI program under Grant no. N0001413-1-0631.

${ }^{1}$ J. Rathsam and L. M. Wang, "A review of diffuse reflections in architecture acoustics," in Proceedings of the 2006 Architectural Engineering National Conference, Building Integration Solutions (AEI, Omaha, NE, 2006), Vol. 23, p. 190.

${ }^{2}$ T. J. Cox and P. D'Antonio, Acoustic Absorbers and Diffuser: Theory, Design and Application, 2nd ed. (Taylor and Francis Group New York, NY, USA, 2009).

${ }^{3}$ N. Yu, P. Genevet, M. A. Kats, F. Aieta, J.-P. Tetienne, F. Capasso, and Z. Gaburro, "Light propagation with phase discontinuities: Generalized laws of reflection and refraction," Science 334, 333-337 (2011).

${ }^{4}$ X. Ni, N. K. Emani, A. V. Kildishev, A. Boltasseva, and V. M. Shalaev, "Broadband light bending with plasmonic nanoantennas," Science 335, 427 (2012).

${ }^{5}$ N. Meinzer, W. L. Barnes, and I. R. Hooper, "Plasmonic meta-atoms and metasurfaces," Nat. Photonics 8, 889-898 (2014).

${ }^{6}$ X. Ni, Z. J. Wong, M. Mrejen, Y. Wang, and X. Zhang, "An ultrathin invisibility skin cloak for visible light," Science 349, 1310-1314 (2015).

${ }^{7}$ D. Lin, P. Fan, E. Hasman, and M. L. Brongersma, "Dielectric gradient metasurface optical elements," Science 345, 298-302 (2014).
${ }^{8}$ M. Khorasaninejad, W. T. Chen, R. C. Devlin, J. Oh, A. Y. Zhu, and F. Capasso, "Metalenses at visible wavelengths: Diffraction-limited focusing and subwavelength resolution imaging," Science 352, 1190-1194 (2016).

${ }^{9}$ X. Ni, A. V. Kildishev, and V. M. Shalaev, "Metasurface holograms for visible light," Nature Commun. 4, 2807 (2013).

${ }^{10}$ L. Huang, X. Chen, H. Muhlenbern, H. Zhang, S. Chen, B. Bai, Q. Tan, G. Jin, K.-W. Cheah, C.-W. Qiu, J. Li, T. Zentgraf, and S. Zhang, "Threedimensional optical holography using a plasmonic metasurface," Nature Comm 4, 2808 (2013).

${ }^{11} \mathrm{G}$. Zheng, H. Muhlenbernd, M. Kenney, G. Li, T. Zentgraf, and S. Zhang, "Metasurface holograms reaching 80\% efficiency," Nature Nano 10, 308-312 (2015).

${ }^{12} \mathrm{Z}$. Liang and J. Li, "Extreme acoustic metamaterial by coiling up space," Phys. Rev. Lett. 108, 114301 (2012).

${ }^{13}$ Z. Liang, T. Feng, S. Lok, F. Liu, K. B. Ng, C. H. Chan, J. Wang, S. Han, $\mathrm{S}$. Lee, and J. Li, "Space-coiling metamaterials with double negative and conical dispersion," Sci. Rep. 3, 1614 (2013).

${ }^{14}$ Y. Xie, B.-I. Popa, L. Zigoneanu, and S. A. Cummer, "Measurement of broadband negative index with space-coiling acoustic metamaterials," Phys. Rev. Lett. 110, 175501 (2013).

${ }^{15}$ S. K. Maurya, A. Pandey, S. Shukla, and S. Saxena, "Double negativity in 3D coiling metamaterials,” Sci. Rep. 6, 33683 (2016).

${ }^{16}$ Y. Li, B. Liang, X. Tao, X. Zhu, X. Zou, and J. Cheng, "Acoustic focusing by coiling up space,” Appl. Phys. Lett. 101, 233508 (2012).

${ }^{17}$ Y. Li, B. Liang, X. Zou, and J. Cheng, "Extraordinary acoustic transmission through ultrathin acoustic metamaterials by coiling up space," Appl. Phys. Lett. 103, 063509 (2013).

${ }^{18}$ Y. Li, X. Jiang, R. Li, B. Liang, X. Zou, L. Yin, and J. Cheng, "Experimental realization of full control of reflected waves with subwavelength acoustic metasurfaces," Phys. Rev. Appl. 2, 064002 (2014).

${ }^{19}$ Y. Xie, W. Wang, H. Chen, A. Konneker, B.-I. Popa, and S. A. Cummer, "Wavefront modulation and subwavelength diffractive acoustics with an acoustic metasurface," Nature Commun. 5, 5553 (2014).

${ }^{20}$ C. Shen, Y. Xie, J. Li, S. A. Cummer, and Y. Jing, "Asymmetric acoustic transmission through near-zero-index and gradient-index metasurfaces," Appl. Phys. Lett 108, 223502 (2016).

${ }^{21}$ K. Song, J. Kim, S. Hur, J.-H. Kwak, S.-H. Lee, and T. Kim, "Directional reflective surface formed via gradient-impeding acoustic meta-surfaces," Sci. Rep. 6, 32300 (2016).

${ }^{22}$ G. P. Ward, R. K. Lovelock, A. R. J. Murray, A. P. Hibbins, J. R. Sambles, and J. D. Smith, "Boundary-layer effects on acoustic transmission through narrow slit cavitites," Phys. Rev. Lett. 115, 044302 (2015).

${ }^{23}$ M. Yang, G. Ma, Z. Yang, and P. Sheng, "Coupled membranes with doubly negative mass density and bulk modulus," Phys. Rev. Lett 110, 134301 (2013).

${ }^{24}$ G. Ma, M. Yang, S. Xiao, Z. Yang, and P. Sheng, "Acoustic metasurface with hybrid resonances," Nature Mater. 13, 873-878 (2014).

${ }^{25}$ S. Zhai, H. Cheng, C. Ding, L. Li, F. Shen, C. Luo, and X. Zhao, "Ultrathin skin cloaks with metasurfaces for audible sound," J. Phys. D: Appl. Phys. 49, 225302 (2016).

${ }^{26}$ H. Esfahlani, S. Karkar, H. Lissek, and J. R. Mosig, "Acoustic carpet cloak based on an ultrathin metasurface," Phys Rev. B 94, 014302 (2016).

${ }^{27}$ C. Faure, O. Richoux, S. Felix, and V. Pagneux, "Experiments on metasurface carpet cloaking for audible acoustics," Appl. Phys. Lett 108, 064103 (2016).

${ }^{28}$ Y. Xie, C. Shen, W. Wang, J. Li, D. Suo, B. I. Popa, Y. Jing, and S. Cummer, "Acoustic holographic rendering with two-dimensional metamaterial based passive phased array," Sci. Rep. 6, 35437 (2016).

${ }^{29}$ K. Melde, A. G. Mark, T. Tian Qiu, and P. Fischer, "Holograms for acoustics," Nature 537, 518-522 (2016). 